\title{
Electrophoretic analysis of sequence variability in three mitochondrial DNA regions for ascaridoid parasites of human and animal health significance.
}

\begin{abstract}
Sequence variability in three mitochondrial DNA (mtDNA) regions, namely cytochrome c oxidase subunit 1 (cox1), NADH dehydrogenase subunits 1 and 4 (nad1 and nad4), among and within Toxocara canis, T. cati, T. malaysiensis, T. vitulorum and Toxascaris leonina from different geographical origins was examined by a mutation-scanning approach. A portion of the cox 1 gene (pcox1), a portion of the nad1 and nad4 genes (pnad1 and pnad4) were amplified separately from individual ascaridoid nematodes by polymerase chain reaction and the amplicons analyzed by single-strand conformation polymorphism (SSCP). Representative samples displaying sequence variation in SSCP profiles were subjected to sequencing in order to define genetic markers for their specific identification and differentiation. While the intra-specific sequence variations within each of the five ascaridoid species were $0.2-3.7 \%$ for pcox $1,0-2.8 \%$ for pnad1 and $0-2.3 \%$ for pnad4, the inter-specific sequence differences were significantly higher, being $7.9-12.9 \%$ for pcox $1,10.7-21.1 \%$ for pnad 1 and $12.9-21.7 \%$ for pnad4, respectively. Phylogenetic analyses based on the combined sequences of pcox 1 , pnad1 and pnad4 revealed that the recently described species T. malaysiensis was more closely related to $T$. cati than to $T$. canis. These findings provided mtDNA evidence for the validity of T. malaysiensis and also demonstrated clearly the usefulness and attributes of the mutation-scanning sequencing approach for studying the population genetic structures of these and other nematodes of socio-economic importance.
\end{abstract}

Keyword: Genetic variation; Mitochondrial DNA (mtDNA); Single-strand conformation polymorphism (SSCP); Toxascaris; Toxocara. 